\title{
Human papillomavirus in head and neck squamous cell carcinoma: A descriptive study of histologically confirmed cases at Kamuzu Central Hospital in Lilongwe, Malawi
}

Claire E. Faggons ${ }^{1}$, Charles E. Mabedi ${ }^{2}$, N. George Liomba ${ }^{1}$, William K. Funkhouser ${ }^{3}$, Fred Chimzimu ${ }^{1}$, Coxcilly Kampani $^{1}$, Robert Krysiak ${ }^{1}$, Nelson Msiska ${ }^{2}$, Carol G. Shores*4,5, Satish Gopal*1,5,6,7

1. UNC Project-Malawi, Lilongwe, Malawi

2. Department of Surgery, Kamuzu Central Hospital, Lilongwe, Malawi

3. Department of Pathology and Laboratory Medicine, University of North Carolina, Chapel Hill, North Carolina, USA

4. Department of Otolaryngology/Head and Neck Surgery, University of North Carolina, Chapel Hill, North Carolinea, USA

5. Lineberger Comprehensive Cancer Center, Chapel Hill, North Carolina, USA

6. Institute for Global Health and Infectious Diseases, University of North Carolina, Chapel Hill, North Carolina, USA

7. Department of Medicine, College of Medicine, University of Malawi, Blantyre, Malawi

*Contributed equally to the article

Correspondence: Dr Satish Gopal (gopal@med.unc.edu)

\section{Background}

\section{Abstract}

Head and neck squamous cell carcinoma (HNSCC) is common in sub-Saharan Africa, but the aetiologic contribution of human papillomavirus (HPV) is not well established.

Methods

We assessed HNSCC cases for HPV using p16 immunohistochemistry (IHC) in Malawi. Associations between p16 IHC and tumour site, behavioural risk factors, demographic characteristics, and HIV status were examined.

Results

From 2010 to 2014, 77 HNSCC cases were identified. Mean age was 52 years, 50 cases (65\%) were male, and 48 (62\%) were in the oropharynx (OP) or oral cavity (OC). HIV status was known for 35 patients (45\%), with 5 (14\%) HIV-infected. Substance use was known for 40 patients (52\%), with 38\% reporting any tobacco and 31\% any alcohol. Forty-two cases (55\%) had adequate tissue for p16 IHC, of which seven $(17 \%)$ were positive, including $22 \%$ of OP/OC tumours.

Conclusions

Despite high cervical cancer burden, HPV-associated HNSCC is not very common in Malawi.

\section{Introduction}

Cancer burden is increasing in sub-Saharan Africa, where one-third of cancers are estimated to be caused by infectious agents. ${ }^{1,2}$ Head and neck squamous cell cancer (HNSCC) is the sixth most common malignancy in sub-Saharan Africa, including tumours in the oral cavity (OC), oropharynx $(\mathrm{OP})$, nasopharynx, other pharynx, and larynx. ${ }^{1}$ Tobacco and alcohol exposure are established risk factors. However, human papillomavirus (HPV) is also a known cause particularly for OP cancer, especially in patients without tobacco or alcohol use. Incidence of HPV-associated OP SCC is increasing in high-income countries, and HPV now accounts for more than $70 \%$ of OP SCC compared to 20 $25 \%$ of HNSCC at other sites. ${ }^{3-5}$

HPV is the causative agent of cervical cancer, for which burden is extremely high throughout sub-Saharan Africa. ${ }^{1}$ However, the contribution of HPV to HNSCC in the region is largely unknown. Prior studies have used varying methodologies to detect HPV [immunohistochemistry (IHC), polymerase chain reaction (PCR), or a combination], often without detailed characterisation of anatomic site or simultaneous evaluation of other risk factors including HIV infection. ${ }^{6-9}$ We therefore sought to determine the presence of HPV using p16 IHC among histologically confirmed
HNSCC cases at Kamuzu Central Hospital (KCH), a national teaching hospital in Malawi's capital, Lilongwe.

\section{Methods}

Histologically confirmed HNSCC cases diagnosed at $\mathrm{KCH}$ between September 2010 and April 2014 were studied. HNSCC was diagnosed using haematoxylin and eosin staining of formalin-fixed paraffin-embedded (FFPE) tissue. All diagnoses were independently confirmed by senior pathologists in Malawi (NGL) and the US (WKF). Review by US pathologists was facilitated by the Aperio virtual microscopy system.10 Expression of p16 was visually assessed by IHC using five micron FFPE sections and the Roche CINtec Histology Kit per manufacturer specifications (Roche Products Ltd, Randburg, South Africa). All sectioning, staining, and IHC was performed manually in Malawi. Both study pathologists independently graded all specimens as negative (no p16 staining), intermediate (weak p16 staining in a minority of cells), or positive (strong p16 staining in a majority of cells). Only specimens considered positive by both pathologists were considered positive in these analyses.

Demographic and clinical data, including HIV status, anatomic site, and substance use, were obtained from medical records. Data were compiled using a standardised 
Table 1: Characteristics of 77 confirmed head and neck squamous cell carcinoma cases in Lilongwe, Malawi

\section{Characteristic}

\begin{tabular}{lc}
\hline Male, n (\%) & $50(65 \%)$ \\
Age, mean years (SD) & $52.4(16.8)$ \\
Tumour site, n (\%) & \\
Oropharynx/oral cavity & $48(62 \%)$ \\
Unknown primary & $13(17 \%)$ \\
Larynx & $9(12 \%)$ \\
Nasopharynx & $6(8 \%)$ \\
Hypopharynx & $1(1 \%)$ \\
HIV status, n (\%) & \\
Positive & $5(6 \%)$ \\
Negative & $30(39 \%)$ \\
Unknown & $42(55 \%)$
\end{tabular}

Tobacco use, $\mathbf{n}(\%)$

Any

None

Unknown

Alcohol use, $\mathbf{n}(\%)$

$\begin{array}{ll}\text { Any } & 12(16 \%) \\ \text { None } & 27(35 \%) \\ \text { Unknown } & 38(49 \%)\end{array}$

$\mathrm{SD}=$ standard deviation

abstraction template. OP/OC sites were grouped together, since most patients presented with bulky tumours making it difficult to definitively assign tumour origin as OP or OC. In addition, some patients were identified via the pathology database alone without baseline assessment by dedicated study clinicians. Anatomic site was therefore often not well characterised for these patients.

Descriptive statistics were summarised and associations between variables determined using a Fisher's exact test and t-test. All data were analysed using Stata 12 (StataCorp, College Station, Texas, USA). The study was approved by the Malawi National Health Science Research Committee and Institutional Review Board of the University of North Carolina.

\section{Results}

Histologically confirmed HNSCC cases diagnosed at $\mathrm{KCH}$ between September 2010 and April 2014 were studied. HNSCC was diagnosed using haematoxylin and eosin staining of formalin-fixed paraffin-embedded (FFPE) tissue. All diagnoses were independently confirmed by senior pathologists in Malawi (NGL) and the US (WKF). Review by US pathologists was facilitated by the Aperio virtual microscopy system. ${ }^{10}$ Expression of p16 was visually assessed by IHC using five micron FFPE sections and the Roche CINtec Histology Kit per manufacturer specifications
Table 2: Characteristics by p16 immunohistochemistry status for 42 head and neck squamous cell carcinoma cases in Lilongwe, Malawi

\begin{tabular}{|c|c|c|c|}
\hline Characteristic & $\begin{array}{c}\text { p16 } \\
\text { positive } \\
(\mathbf{n}=7)\end{array}$ & $\begin{array}{c}\text { p16 } \\
\text { negative } \\
(n=35)\end{array}$ & P-value \\
\hline Male, n (\%) & 4 & 23 & 0.68 \\
\hline Age, mean years (SD) & $49.1(8.3)$ & $54.3(18.0)$ & 0.47 \\
\hline Oropharynx/oral cavity, n (\%) & $5(71 \%)$ & $18(51 \%)$ & 0.43 \\
\hline \multicolumn{4}{|l|}{ HIV status, n (\%) } \\
\hline Positive & $1(14 \%)$ & $1(3 \%)$ & 0.43 \\
\hline Negative & $3(43 \%)$ & $12(34 \%)$ & \\
\hline Unknown & $3(43 \%)$ & $22(63 \%)$ & \\
\hline Tobacco use, n (\%) & & & - \\
\hline Any & $0(0 \%)$ & $3(9 \%)$ & \\
\hline None & $1(14 \%)$ & $3(9 \%)$ & \\
\hline Unknown & $6(86 \%)$ & $29(83 \%)$ & \\
\hline Alcohol use, n (\%) & & & - \\
\hline Any & $0(0 \%)$ & $0(0 \%)$ & \\
\hline None & $1(14 \%)$ & $6(17 \%)$ & \\
\hline Unknown & $6(86 \%)$ & $29(83 \%)$ & \\
\hline
\end{tabular}

(Roche Products Ltd, Randburg, South Africa). All sectioning, staining, and IHC was performed manually in Malawi. Both study pathologists independently graded all specimens as negative (no p16 staining), intermediate (weak p16 staining in a minority of cells), or positive (strong p16 staining in a majority of cells). Only specimens considered positive by both pathologists were considered positive in these analyses. Demographic and clinical data, including HIV status, anatomic site, and substance use, were obtained from medical records. Data were compiled using a standardised abstraction template. OP/OC sites were grouped together, since most patients presented with bulky tumours making it difficult to definitively assign tumour origin as OP or OC. In addition, some patients were identified via the pathology database alone without baseline assessment by dedicated study clinicians. Anatomic site was therefore often not well characterised for these patients.

Descriptive statistics were summarised and associations between variables determined using a Fisher's exact test and t-test. All data were analysed using Stata 12 (StataCorp, College Station, Texas, USA). The study was approved by the Malawi National Health Science Research Committee and Institutional Review Board of the University of North Carolina.

\section{Discussion}

Despite a concentration of infection-associated cancers in sub-Saharan Africa including an immense burden of HPVassociated cervical cancer, ${ }^{11}$ and rising incidence of HPVassociated OP SCC in high-income countries, ${ }^{3-5}$ we found few HPV-associated HNSCC specimens at a national teaching hospital in Malawi as assessed by p16 IHC. Our study was limited by small sample size, as well as missing data, due to reliance on routine medical records for abstracting HIV status and clinical information. Nevertheless, these results may be valuable in light of few studies specifically examining 
Table 3: p16 immunohistochemistry status by anatomic site for $\mathbf{4 2}$ head and neck squamous cell carcinoma cases in Lilongwe, Malawi

\begin{tabular}{lccc}
\hline \multirow{2}{*}{ Tumour site } & \multicolumn{2}{c}{ Total } & \multicolumn{2}{c}{$\mathbf{p 1 6}$ positive } \\
\cline { 3 - 4 } & & $\mathbf{n}$ & $\%$ \\
\hline Oropharynx/oral cavity & 23 & 5 & $22 \%$ \\
Unknown primary & 8 & 0 & $0 \%$ \\
Larynx & 6 & 2 & $33 \%$ \\
Nasopharynx & 4 & 0 & $0 \%$ \\
Hypopharynx & 1 & 0 & $0 \%$ \\
All sites & 42 & 7 & $17 \%$ \\
\hline
\end{tabular}

HPV-associated HNSCC in sub-Saharan Africa, and no prior studies in Malawi to our knowledge.

Despite its limitations, our results are generally consistent with the few other contemporary studies existing from the region. A recent study from Mozambique found no HPVassociated tumours among $51 \mathrm{OP} / \mathrm{OC}$ cases using p16 IHC as well as PCR detection of E6 and E7 oncogene products. ${ }^{9}$ Similarly, a study from Senegal found 3\% HPV DNA detection by PCR among 117 HNSCC cases, with no PCR-positive cases being positive by p16 $\mathrm{IHC}^{7}$ Other studies from Sudan and South Africa have detected HPV DNA by PCR in $27-49 \%$ of OP/OC tumours. ${ }^{6,8}$ However, PCR-based detection alone can often identify bystander oral HPV that may not be causally implicated in oncogenesis, a process for which p16 IHC is a more reliable surrogate. ${ }^{5}$ Our findings are also consistent with global epidemiologic data suggesting that increases in OP cancer, the HNSCC site which has the closest causal association with HPV, are largely confined to more economically developed countries rather than less developed countries. ${ }^{4,5}$

If these data are collectively correct in suggesting low frequency for HPV-associated HNSCC in sub-Saharan Africa, this is despite an extremely high burden of HPV-associated cervical cancer, ${ }^{11}$ including in Malawi specifically. ${ }^{12-14}$ While this is speculative, one reason for this discrepancy may be differences in sexual practices, which are major determinants of oral HPV infection and HPV-associated HNSCC risk., ${ }^{5}$ Such data are absent from Malawi and were not specifically assessed in our study, but other regional literature have suggested that oral intercourse and oral HPV infection are uncommon at least in South Africa. ${ }^{16}$

In our study, although HIV status was missing for many patients thereby introducing potential bias, we found an HIV prevalence of $14 \%$ among cases with known HIV status, roughly similar to $10-11 \%$ prevalence in the Malawi general population. ${ }^{17}$ Although HIV is associated with an approximately 2-3-fold increase in HNSCC risk in resource-rich settings, ${ }^{18,19}$ our data do not suggest a major association between HIV and HNSCC in Malawi, a country with high HIV prevalence. This may reflect generally low rates of tobacco and alcohol use in our setting (14\% and $7 \%$ in the Malawi general population respectively). ${ }^{20}$ Among HIV-infected persons in resource-rich settings, higher rates of tobacco and alcohol use and HPV contribute to increased HNSCC compared to HIV-negative people. ${ }^{18,19}$ Also, despite remarkable progress in antiretroviral therapy (ART) scale-up, coverage is still lower in Malawi than high- income countries with typically more advanced HIV illness prior to ART initiation. Therefore, Malawi may have still to realise epidemiologic transitions in its HIV-infected population that have occurred in other countries, where opportunistic infections and classical AIDS-defining cancers have been supplanted by non-AIDS-defining cancers and cardiovascular disease as principal causes of morbidity and mortality, among aging HIV-infected populations effectively treated with ART.

In summary, HPV-associated HNSCC as assessed by p16 IHC was uncommon in our small series at a national teaching hospital in Malawi. Continued HNSCC surveillance in Malawi will be important to fully understand the importance of this disease moving forward, ideally through larger regional collaborations across sub-Saharan Africa which can provide more definitive results. This will be particularly important as ART coverage increases for HIV-infected individuals, behavioural practices change, and HPV vaccination becomes available. Finally, if established risk factors including tobacco, alcohol, and HPV do not collectively account for most HNSCC in sub-Saharan Africa, high-quality epidemiologic studies are urgently needed to identify aetiologic agents in HNSCC development which may be unique to the region. These vital data can inform prevention efforts in settings where treating established HNSCC is difficult due to resource limitations, including exceedingly scarce radiotherapy.

\section{Acknowledgements}

C.E.F. was supported by the UJMT Fogarty Global Health Fellows Program (R25TW009340). S.G. is supported by grants from the National Institutes of Health (K01TW009488, R21CA180815, and U54CA190152) and the AIDS Malignancy Consortium (U01CA121947). This work was also supported by the Medical Education Partnership Initiative (U2GPS001965) and Lineberger Comprehensive Cancer Center (P30CA016086). We additionally acknowledge leadership of Kamuzu Central Hospital (Jonathan Ngoma), UNC Project-Malawi (Irving Hoffman, Innocent Mofolo, Mina Hosseinipour, Francis Martinson), Lineberger Comprehensive Cancer Center (Shelley Earp, Ned Sharpless, Lisa Carey, Blossom Damania, Dirk Dittmer), and Malawi Surgical Initiative (Anthony Charles) for their support of this study.

\section{Competing interests}

All authors declare that they have no competing interests related to this work.

\section{References}

1. Ferlay J, Soerjomataram I, Ervik M, et al (2013) GLOBOCAN 2012 v1.0, Cancer Incidence and Mortality Worldwide: IARC CancerBase No. 11. Lyon, France: International Agency for Research on Cancer. http://globocan.iarc.fr. Accessed February 9, 2016

2. de Martel C, Ferlay J, Franceschi S, et al (2012) Global burden of cancers attributable to infections in 2008: a review and synthetic analysis. Lancet Oncol 13:607-615.

3. Chaturvedi AK, Engels EA, Pfeiffer RM, et al (2011) Human papillomavirus and rising oropharyngeal cancer incidence in the United States. J Clin Oncol 29:4294-4301.

4. Chaturvedi AK, Anderson WF, Lortet-Tieulent J, et al (2013) Worldwide trends in incidence rates for oral cavity and oropharyngeal cancers. J Clin Oncol 31:4550-4559.

5. Gillison ML, Chaturvedi AK, Anderson WF, et al (2015) Epidemiology of Human Papillomavirus-Positive Head and Neck Squamous Cell Carcinoma. J Clin Oncol 33:3235-3242. 
6. Jalouli J, Ibrahim SO, Sapkota D, et al (2010) Presence of human papilloma virus, herpes simplex virus and Epstein-Barr virus DNA in oral biopsies from Sudanese patients with regard to toombak use. J Oral Pathol Med 39:599-604.

7. Ndiaye C, Alemany L, Diop Y, et al (2013) The role of human papillomavirus in head and neck cancer in Senegal. Infect Agent Cancer 8:14.

8. Paquette C, Evans MF, Meer SS, et al (2013) Evidence that alpha-9 human papillomavirus infections are a major etiologic factor for oropharyngeal carcinoma in black South Africans. Head Neck Pathol 7:361-372.

9. Blumberg J, Monjane L, Prasad M, et al (2015) Investigation of the presence of HPV related oropharyngeal and oral tongue squamous cell carcinoma in Mozambique. Cancer Epidemiol 39:1000-1005.

10. Gopal S, Krysiak R, Liomba NG, et al (2013) Early experience after developing a pathology laboratory in Malawi, with emphasis on cancer diagnoses. PLoS One 8:e70361.

11. De Vuyst H, Alemany L, Lacey C, et al (2013) The burden of human papillomavirus infections and related diseases in sub-saharan Africa. Vaccine 31 Suppl 5:F32-46.

12. Motti PG, Dallabetta GA, Daniel RW, et al (1996) Cervical abnormalities, human papillomavirus, and human immunodeficiency virus infections in women in Malawi. J Infect Dis 173:714-717.

13. Reddy D, Njala J, Stocker P, et al (2015) High-risk human papillomavirus in HIV-infected women undergoing cervical cancer screening in Lilongwe, Malawi: a pilot study. Int J STD AIDS 26:379387.
14. Msyamboza KP, Dzamalala C, Mdokwe C, et al (2012) Burden of cancer in Malawi; common types, incidence and trends: national population-based cancer registry. BMC Res Notes 5:149.

15. Gillison ML, Castellsague X, Chaturvedi A, et al (2014) Eurogin Roadmap: comparative epidemiology of HPV infection and associated cancers of the head and neck and cervix. Int J Cancer 134:497-507.

16. Davidson CL, Richter KL, Van der Linde M, et al (2014) Prevalence of oral and oropharyngeal human papillomavirus in a sample of South African men: a pilot study. S Afr Med J 104:358-361.

17. UNAIDS. Malawi Progress Report for 2015. http://www.unaids. org/en/regionscountries/countries/malawi. Accessed February 9, 2016

18. D'Souza G, Carey TE, William WN, Jr., et al (2014) Epidemiology of head and neck squamous cell cancer among HIV-infected patients. J Acquir Immune Defic Syndr 65:603-610.

19. Beachler DC, Abraham AG, Silverberg MJ, et al (2014) Incidence and risk factors of HPV-related and HPV-unrelated Head and Neck Squamous Cell Carcinoma in HIV-infected individuals. Oral Oncol 50:1169-1176.

20. Msyamboza KP, Ngwira B, Dzowela T, et al (2011) The burden of selected chronic non-communicable diseases and their risk factors in Malawi: nationwide STEPS survey. PLoS One 6:e20316. 